These aspects of policy making are often implicit and unstated except when economists point them out, to the unease of decision makers.

Evidence is gathered and organised by economists, epidemiologists, and public health professionals. The needs of the population are usually described by public health professionals, but they are often hindered by the lack of programme budgeting and the dearth of good quality information about the incidence or prevalence of disease in a local population.

Values, however, are for the public to decide or more usually by their elected representatives. This is a source of annoyance to professionals whose proposal is not funded because of a political decision, but this annoyance is usually because the politician is operating to a different set of values than the professional whose values may be based just as much on emotion as the politician's values.

What can a professional do in this context? The job of the professional or technician is to set out all the information about the probability and size of benefits and harms, and about the opportunity costs, namely the other uses that could be made of the same amount of resources, taking into account the needs of the population. This is decision making. The job of the politician is to take the decision, as opposed to making the decision, based on values. In the end values will always be more influential than evidence, and the tension between the two should be regarded as the very stuff of the relationship between expert and politician.

\section{J A Muir Gray programme director, UK National Screening Committee}

Institute of Health Sciences, University of Oxford, Oxford OX3 7LF (muir.gray@ihs.ox.ac.uk)

1 Wald NJ, Rodeck C, Hackshaw AK, Walters J, Chitty L, Mackinson AM. First and second trimester antenatal screening for Down's syndrome: the results of the serum, urine and ultrasound screening study (SURUSS). results of the serum, urine and
Health Technol Assess 2003;7:11.

2 Alfirevic $Z$ and Neilson JP. Antenatal screening for Down's syndrome. BMJ 2004;329:811-2.

\title{
Teaching evidence based medicine
}

\section{Should be integrated into current clinical scenarios}

$\mathrm{T}$ Teaching clinical epidemiology has always been challenging, seen as too mathematical and remote from normal clinical practice. Evidence based medicine (EBM) evolved to provide the skills needed to manage the potential information overload of modern medical schools, especially at McMaster, the shortest medical programme in the world. Students have to grasp two essential principles of EBM: its empirical approach to optimal clinical decisions (regardless of pathophysiology, does the bottom line of the balance sheet show gain or loss?); and its quantitative expression (how big is that gain or loss?). These require some mastery of epidemiology and statistics, both repellent to many doctors, even in teaching hospitals. ${ }^{12}$ Now most medical programmes in the United States attempt to teach EBM, although few succeed (the two most important barriers being inadequate access to electronic information at the point of care, and inadequate faculty training). ${ }^{3}$

What are the best ways of teaching this stuff? By breaking its elements into manageable chunks-asking, accessing, appraising, applying. Often a fifth element is added-assessing (box).

Every mode of delivery has been used: lectures, mini-courses; tutorials to help students work through problems; books to do it alone or as an accompaniment to a course ${ }^{4}$; and even subscription websites.

Education of medical students in EBM ranges from passing mention to multi-year courses of over 100 hours. For example, Albany Medical College has a four year compulsory pass-fail EBM course lasting 120 hours over four years. It uses lectures, small group sessions, and written assignments to teach students critical appraisal and clinical decision making and then apply them to various exercises during the clinical years in each of their clinical clerkships. Its evaluation shows of when it is not being taught well in other parts of the programme, and an improvement in biostatistics and epidemiology performance on standardised examinations.

Less conventional forms of teaching include critically appraised topics, which develop and assess the range of EBM skills. Students pose a clinical question and answer it, presenting the critically appraised topic to their teachers and peer group for grading, (for example, the Sydney PEARLs programme (www.gmp.usyd.edu.au/vguide/educators/ ie_features_ebm.html) and the postgraduate Manchester BestBets (www.bestbets.org)). Important markers of success may be: questions initiated by students; brief presentations; small groups; multiple clinical settings; and evaluation. Occasionally these questions and answers have been interesting enough to lead to worthwhile publications and even Cochrane reviews. ${ }^{6-8}$ Unlike the common and time consuming research projects this exercise focuses on (future) clinicians as users of research rather than producers of it.

\section{Elements of evidence based learning}

- Asking-converting the clinical puzzle into an answerable question

- Accessing-searching to find the answer to that question

- Appraising-critically evaluating the evidence to decide if it is, and if so how, reliable and robust

- Applying-extracting the useful information and addressing the thorny issues of generalisability and "particularis-ability" to decide what clinical action is best

Often a fifth element is added

- Assessing-evaluation of the process to integrate this element into the quality improvement cycle 
What evidence is there that teaching EBM achieves its aims? A five year old Cochrane review found sparse evidence ${ }^{9}$-one randomised controlled trial showing that about six hours of journal club time devoted to critical appraisal increased knowledge of this. Two subsequent randomised controlled trials with broader teaching showed a sustained educational benefit across several of the processes of EBM..$^{10}{ }^{11}$ A systematic review in this issue shows that integrating the teaching of the steps of EBM with clinical practice is vital to improving attitudes, skills, and behaviour. ${ }^{12}$ Integration means applying the steps to real and current clinical problems. Thinking is not enough and doing is necessary for success.

Additionally, role modelling may be necessary. ${ }^{13}$ Unless students see their role models use EBM in practice, they are unlikely to value it as clinically important. Therefore, specific content in their daily clinical education must refer to relevant trials and cohort studies to show how research methods integrate with clinical practice. Teaching EBM may need to focus as much on teachers as on students and registrars.

In postgraduate environments one useful modelling step is modified and question driven journal clubs to enable registrars in hospital or general practice to engage in a communal EBM activity. ${ }^{14}$ Another way to improve the teaching of EBM in the postgraduate environment is to create evidence teams consisting of registrars and medical students to find evidence in everyday clinical settings. Their evaluation of the evidence can then be evaluated by the consultant on the team, who would be in the best position to evaluate the use of that evidence for the patient.

EBM is here to stay. It has become an essential way of teaching and practising in the uncertain world of medicine. The challenge is to engage the whole healthcare team in learning about it and making it part of the routine of clinical practice.

\section{Chris Del Mar dean}

Faculty of Health Science and Medicine, Bond University, Qld 4229, Australia

(CDelMar@staff.bond.edu.au)

Paul Glasziou director

Centre for Evidence-Based Practice, Oxford OX3 7LF

Dan Mayer professor of emergency medicine

Albany Medical College, 47 New Scotland Avenue, Albany, NY 12208 USA

Competing interests: None declared.

1 Godwin M, Seguin R. Critical appraisal skills of family physicians in Ontario, Canada. BMC Med Educ 2003:3:10.

2 Young JM, Glasziou P, Ward JE. General practitioners' self ratings of skills in evidence based medicine: validation study. BMJ 2002;324:950-1.

in evidence based medicine: validation study. BMJ 2002;324:950-1.
Green ML. Evidence-based medicine training in internal medicine Green ML. Evidence-based medicine training in internal medicine
residency programs a national survey.J Gen Intern Med 2000;15:129-33.

4 Glasziou P, Del Mar C, Salisbury J. Evidence-based medicine workbook. Finding and applying the best research evidence to improve patient care. 1st ed. London: BMJ Books, 2003.

5 Mayer D. Essential evidence-based medicine. Cambridge: Cambridge University Press, 2004.

6 Allen C, Glasziou P, Del Mar C. Bed rest: a potentially harmful treatment needing more careful evaluation. Lancet 1999;354:1229-33.

7 Thomas M, Del Mar C, Glasziou P. How effective are treatments other than antibiotics for acute sore throat? Br J Gen Pract 2000;50:817-20.

8 Francis D, Del Mar C, Thomas M, Glasziou P. Non-antibiotic treatments for sore throat (protocol for a Cochrane review). Cochrane Library. for sore throat (protocol for
Chichester: John Wiley, 2004.

9 Parkes J, Hyde C, Deeks J, Milne R. Teaching critical appraisal skills in health care settings [Cochrane review]. Cochrane Library. Chichester:John Wiley, 2004.

10 Smith CA, Ganschow PS, Reilly BM, Evans AT, McNutt RA, Osei A, et al. Teaching residents evidence-based medicine skills: a controlled trial of effectiveness and assessment of durability. J Gen Intern Med 2000;15:710-5.

11 Green ML, Ellis PJ. Impact of an evidence-based medicine curriculum based on adult learning theory.J Gen Intern Med 1997;12:742-50.

12 Coomarasamy A, Khan KS. What's the evidence that postgraduate teachCoomarasamy A, Khan KS. What's the evidence that postgraduate teach-
ing in evidence-based medicine changes anything? A systematic review.

13 Sackett DL, Straus SE, Richardson WS, Rosenberg W, Haynes RB. Evidence-based medicine. How to practise and teach EBM. 2nd ed. Edinburgh: Churchill Livingstone, 2000.

14 Del Mar CB, Glasziou PP. Ways of using evidence-based medicine in general practice. Med J Aust 2001;174:347-50

\section{Evidence based medicine has come a long way}

\section{The second decade will be as exciting as the first}

$\mathrm{E}$ vidence based medicine seeks to empower clinicians so that they can develop independent views regarding medical claims and controversies. Although many helped to lay the foundations of evidence based medicine ${ }^{1}$ Archie Cochrane's insistence that clinical disciplines summarise evidence concerning their practices, Alvan Feinstein's role in defining the principles of quantitative clinical reasoning, and David Sackett's innovation in teaching critical appraisal all proved seminal. The term evidence based medicine, ${ }^{2}$ and the first comprehensive description of its tenets, appeared little more than a decade ago. In its original formulation, this discipline reduced the emphasis on unsystematic clinical experience and pathophysiological rationale, and promoted the examination of evidence from clinical research. Evidence based medicine therefore required new skills including efficient literature searching and the application of formal rules of evidence in evaluating the clinical literature.

Important developments in evidence based medicine over the subsequent decade included the increas- ing popularity of structured abstracts ${ }^{3}$ and secondary journals summarising studies of high relevance and methodological quality, ${ }^{4}$ the creation of the Cochrane Collaboration and its systematic reviews, and the publication of innovative medical texts emphasising evidence based decision making. The principles of evidence based medicine have become core concepts of undergraduate, postgraduate, and continuing medical education, and courses, workshops, and online resources have proliferated.

The philosophy of evidence based medicine has evolved. Exponents increasingly emphasise the limitations of using evidence alone to make decisions, and the importance of the values and preference judgments that are implicit in every clinical management decision. They now see clinical expertise as the ability to integrate research evidence and patients' circumstances and preferences to help patients arrive at optimal decisions.

Evidence based medicine, still young, faces challenges in integration into clinical practice. The process of producing relevant evidence through high 